\title{
Hartmut Lehmann, Jean-Martin Ouedraogo, éds., Max Webers Religionssoziologie in interkultureller Perspektive
}

Göttingen, Vandenhoeck \& Ruprecht, coll. «Veröffentlichungen des Max-Planck-Institus für Geschichte und Gesellschaft », 2003, 388 p.

Jean-Louis Schlegel

\section{(Q) OpenEdition}

\section{Journals}

Édition électronique

URL : http://journals.openedition.org/assr/3187

DOI : $10.4000 /$ assr.3187

ISSN : $1777-5825$

Éditeur

Éditions de l'EHESS

Édition imprimée

Date de publication : 1 décembre 2005

Pagination : 215-311

ISBN : 2-7132-2045-9

ISSN : 0335-5985

\section{Référence électronique}

Jean-Louis Schlegel, « Hartmut Lehmann, Jean-Martin Ouedraogo, éds., Max Webers

Religionssoziologie in interkultureller Perspektive », Archives de sciences sociales des religions [En ligne]

131-132 | juillet - décembre 2005, document 132-47, mis en ligne le 27 mars 2006, consulté le 21

septembre 2020. URL : http://journals.openedition.org/assr/3187; DOI : https://doi.org/10.4000/assr. 3187

Ce document a été généré automatiquement le 21 septembre 2020.

(C) Archives de sciences sociales des religions 


\section{Hartmut Lehmann, Jean-Martin Ouedraogo, éds., Max Webers Religionssoziologie in interkultureller Perspektive}

Göttingen, Vandenhoeck \& Ruprecht, coll. « Veröffentlichungen des Max-Planck-Institus für Geschichte und Gesellschaft », 2003, 388 p.

Jean-Louis Schlegel

1 En France notamment, la discussion sur Weber a largement porté sur sa vision sociologique générale, sa méthode d'approche des sociétés et des religions, ses rapports avec la philosophie, ses " gros » concepts méthodologiques (religiosité de virtuoses ou de masse, biens et institution du salut, rationalisation de la conduite de la vie, ascèse intramondaine...). Il se peut que les nouvelles et récentes traductions, d'une exceptionnelle fiabilité, de Jean-Pierre Grossein et de quelques autres changent la donne. En tout cas, l'intérêt du colloque franco-allemand tenu à Göttingen en juin 2000 et intitulé « Religion, État et société chez Max Weber. Comparaison interculturelle et bilan critique » vient de ce qu'il considère d'abord des thèmes moins étudiés ou moins larges, comme les sources intellectuelles (éclectiques et partielles) que Weber a utilisées ou ses questions personnelles en rapport avec sa recherche. Surtout, la majeure partie des contributions reprend de manière critique les analyses de Weber sur diverses religions et groupes religieux dans le monde (hindouisme, bouddhisme, confucianisme et taoïsme, catholicisme, luthéranisme, calvinisme, baptistes et quakers, monachisme, ou encore la réception de Weber dans l'orthodoxie russe). Même si le colloque s'est, pour partie, divisé entre ceux qui continuent de voir en Weber un «visionnaire » utile sinon indispensable à la compréhension de nos questions du $\mathrm{XxI}^{\mathrm{e}}$ siècle et ceux qui ont un regard plus critique, plus "contextualisé », on peut considérer ces derniers comme plus incisifs et plus «stimulants » finalement dans leur approche du wébérianisme. On se contentera ici de quelques aspects, glanés dans un volume au contenu, par définition, éclaté. 
Weber, comme on sait, était d'origine luthérienne. Mais s'il admirait Luther lui-même, " une tour qui dépasse toutes les autres [i.e. "Calvin, Fox et tutti quanti", nommément cités dans ce contexte]», il vouait, comme il le reconnaît dans une lettre de 1906 à Harnack, une haine sans bornes aux formes historiques du luthéranisme, "la plus épouvantable des épouvantes", qui a instillé un esprit de soumission à l'État sans équivalent dans les États protestants (cette critique s'est encore accentuée au xxe siècle, après la tragédie nazie - mais un article de Sabine Holtz tente de la rééquilibrer quelque peu). Comme nombre d'autres intellectuels libéraux, marqués par un Kulturprotestantismus désireux d'accorder la «liberté évangélique » et la culture de l'époque moderne, il lutta, après le départ de Bismarck, pour un État démocratique, avec une séparation stricte des religions et de l'État (à cet égard, il était plus nettement "séparateur", semble-t-il, que d'autres, Troeltsch entre autres, qui insistaient en même temps sur les liens historiques et sociologiques entre nations européennes et christianisme). Toujours dans une lettre à Harnack mais en 1909, Weber déclare, assez curieusement mais logiquement, se méfier d'un catholicisme qui (à l'instar du luthéranisme) aimerait désormais se couler dans les moules juridiques de l'État: une «technique d'adaptation », une "élasticité » qui lui « répugnent» (il est question, si nous avons bien compris, de la reconnaissance du mariage catholique par l'État). On appréciera aussi (dans cette même lettre) la sombre prévision suivante : « S'il me faut soupeser les chances pour l'avenir, ce sont, selon moi, deux puissances qui ont les chances théoriques les plus favorables de parvenir à soumettre toutes les autres: le bureaucratisme dans l'État et la machinerie virtuose [sic: die virtuose Maschinerie] de l'Église catholique, en lien avec la parcellisation qui réduit l'humanité à l'homme de la spécialisation (économique et autre).» Combattre cette tendance relève pour lui d'une " exigence de ma dignité humaine ». Sur l'État, le droit, le politique en général, et les concepts qu'il a utilisés (bureaucratie, charisme/fonction...), des intervenants allemands (Gangolf Hübinger et Andreas Anter) rappellent à quel point Weber a été redevable à des auteurs écrivant avant lui ou contemporains, en particulier, pour les rapports entre le politique et la religion, aux travaux de Rudolf Sohm.

3 À propos des considérations wébériennes sur les "religions du monde ", plusieurs contributions insistent sur la faiblesse de sa documentation, son caractère daté, partiel, dépassé par les informations connues au xx $x^{e}$ siècle (ainsi, pour la "ville en islam ", il n'aurait utilisé qu'un seul livre, consacré à La Mecque), ou encore sur une vision marquée par l'époque (ainsi de sa conception «coloniale» de l'Inde). Tout au plus, certains lui reconnaissent-ils le mérite d'avoir posé les « bonnes questions ».

Retenons encore qu'en dépit de l'anticatholicisme ambiant en Allemagne (avec ses clichés, par exemple, sur l'irrationnalité catholique dans les temps modernes - clichés véhiculés dans les milieux luthériens de sa jeunesse et de son âge adulte), Weber n'a jamais varié sur la rationalité, "en finalité "si on veut, du catholicisme, de ses institutions et de son droit, et sur leur importance pour le devenir de l'Occident. Dans l'excellent article de Hartmann Tyrell sur le thème du catholicisme, je retiens aussi ce que chacun de nous sait implicitement : quand Weber parle du type "Église ", c'est bien l'Église catholique qu'il a en tête, mais elle est ce type dans deux perspectives (qui se croisent partiellement) : comme forme de pouvoir (avec ses conséquences pour le droit et le pouvoir laïque en Occident) et comme opposée au type "secte». Selon Tyrell, Weber n'inclinait guère à considérer la primitive Église comme une secte, à l'encontre d'une conviction fort partagée aujourd'hui (et par Tyrell lui-même, qui avance ses 
raisons). Dans un bref chapitre (en anglais, titré «Catholic Ethics and the Spirit of Catholicism »), notre collègue Michaël Löwy traite, en clin d'œil évident, d'une « sorte d'affinité négative, ou d'“antipathie culturelle" entre éthique catholique et esprit du capitalisme ».

5 Sur le calvinisme, sur les baptistes et les quakers américains, la critique des célèbres idées wébériennes est surtout faite à partir des nouvelles connaissances historiques qui se sont imposées depuis L'Éthique protestante. À l'inverse, une importante contribution, de O.G. Oexle sur "Weber et le monachisme", souligne que la recherche historique récente insiste sur l'idée de vita communis et de vie régulée chez les moines, plutôt que sur leur "idéal ascétique», comme l'ont fait longtemps, à la suite de Harnack, les historiens protestants. Or, sur ce point, tout en étant tributaire de Harnack, Weber a été beaucoup moins unilatéral et a insisté sur d'autres valeurs, non ascétiques, de la vie monastique. Le volume se conclut par deux réflexions plus larges, en français, de JeanMartin Ouedraogo - sur les rapports entre «sociologie des religions, sécularisation et agir politique » chez Weber -, et de Catherine Colliot-Thélène, par ailleurs traductrice de Weber («Que faire du comparatisme wébérien?»), qui rappelle entre autres que Weber n'était pas dupe des limites éventuelles de ses sources. À noter encore, auparavant, une contribution intéressante (de Johannes Weiss) sur l'originalité de Weber, qui a maintenu fermement la légitimité propre de la religion et refuse de l'« expliquer » en la référant, comme tant d'approches critiques, à des réalités autres qu'elles-mêmes (la misère, la société, la répression de la vie...). 